\title{
Heat Capacity and Susceptibility of $\mathrm{CeCu}_{4} \mathrm{Al}$
}

\author{
M. Reiffers ${ }^{a}$, A. KowalczyK ${ }^{b}$, T. TOliński $^{b}$,

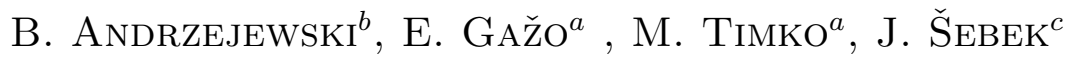 \\ AND E. ŠanTAVÁ ${ }^{c}$ \\ ${ }^{a}$ Institute of Experimental Physics, Slovak Academy of Sciences \\ Watsonova 47, 04353 Košice, Slovakia \\ ${ }^{b}$ Institute of Molecular Physics, Polish Academy of Sciences \\ Smoluchowskiego 17, 60-179 Poznań, Poland \\ ${ }^{c}$ Institute of Physics, Academy of Sciences of the Czech Republic \\ Na Slovance 2, 18221 Praha 8, Czech Republic \\ The heat capacity in the applied magnetic field up to $9 \mathrm{~T}$, susceptibility \\ and magnetization of polycrystalline $\mathrm{CeCu}_{4} \mathrm{Al}$ are presented. The deter- \\ mined electronic heat capacity coefficient $\gamma=210 \mathrm{~mJ} \mathrm{~mol}^{-1} \mathrm{~K}^{-2}$ confirmed \\ heavy fermion character of this compound. Magnetic ordering was not ob- \\ served down to $0.5 \mathrm{~K}$. Magnetic properties confirm these observations.
}

PACS numbers: 75.40.Cx, 75.30.Mb

\section{Introduction}

Ce-intermetallics are always very attractive compounds due to variety of interesting behaviour. They are frequently characterised by the anomalous proximity of the Ce $4 f$-level to the Fermi energy. This proximity yields a strong hybridisation of the magnetic $4 f$ electrons with the conduction electrons. $\mathrm{CeCu}_{4} \mathrm{Al}$ is known as the heavy fermion compound $[1,2]$ and it is the derivate of $\mathrm{CeCu}_{5}$, which undergoes two magnetic transitions below $4 \mathrm{~K}$ indicating complicated magnetic structure. However, $\mathrm{CeCu}_{4} \mathrm{Al}$ does not order magnetically down to the lowest studied temperature. The estimated value of electronic coefficient $\gamma$ was of about $280 \mathrm{~mJ} \mathrm{~mol}^{-1} \mathrm{~K}^{-2}[2]$. Below $6 \mathrm{~K} C / T$ dependence showed a strong increase, which yields the value of $C / T$ of about $1.1 \mathrm{~J} \mathrm{~mol}^{-1} \mathrm{~K}^{-2}$ [2]. The measurements of $\gamma$ in the magnetic fields up to $12 \mathrm{~T}$ showed the reduction of $C / T$ values by a factor 5 at low temperatures [3].

In order to study the magnetic field behaviour of the heavy fermion system $\mathrm{CeCu}_{4} \mathrm{Al}$ at low temperatures we have measured the heat capacity in the applied magnetic field, susceptibility, and magnetisation of the polycrystalline sample. 


\section{Experiment}

The $\mathrm{CeCu}_{4} \mathrm{Al}$ compound was prepared by induction melting of stoichiometric amounts of the constituent elements in a water-cooled boat, under an argon atmosphere. The ingots were inverted and melted several times to ensure homogeneity. The crystal structure was established by a powder X-ray diffraction technique, using $\mathrm{Cu} K_{\alpha}$ radiation. The $\mathrm{CeCu}_{4} \mathrm{Al}$ compound crystallises in the hexagonal $\mathrm{CaCu}_{5}$-type of structure, space group $\mathrm{P} 6 / \mathrm{mmm}$. The lattice constants are $a=5.175 \AA$ and $c=4.148 \AA$.

Heat capacity measurements were performed by PPMS commercial device (Quantum Design) in Košice and Prague, in the temperature range 0.5-300 K by relaxation method using two- $\tau$ model. The magnetic susceptibility and the magnetization curves were measured on the MagLab 2000 System (Oxford Instruments Ltd.).

\section{Results}

Figure 1a shows the temperature dependence of the heat capacity $C(T)$ of $\mathrm{CeCu}_{4} \mathrm{Al}$ in the temperature range $0.5-300 \mathrm{~K}$ and in zero magnetic field. We have not seen any real sign of the magnetic order down to $0.5 \mathrm{~K}$. In order to compare with previous results [1-3] we present in Fig. 1b the low temperature part of $C(T) / T$ behaviour. The extrapolation to $T=0 \mathrm{~K}$ of the temperature range above $11 \mathrm{~K}$ yields a $\gamma$ value of about $210 \mathrm{~mJ} \mathrm{~mol}{ }^{-1} \mathrm{~K}^{-2}$. This value confirms the heavy fermion character of this compound. Extrapolation of the lowest temperatures range of $C / T\left(T^{2}\right)$ yields the value of $2.2 \mathrm{~J} \mathrm{~mol}^{-1} \mathrm{~K}^{-2}$. The observed behaviour is in a qualitative agreement with Refs. [1,2]. Our magnitudes of $C(T)$ and $\gamma$ are smaller, except the lowest temperature extrapolation.
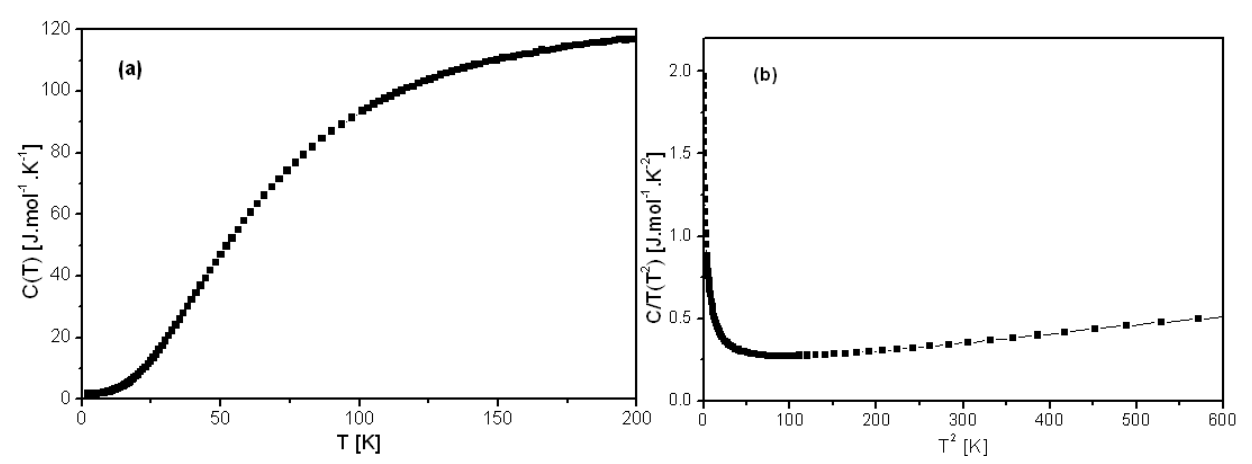

Fig. 1. (a) The heat capacity $C(T)$ of polycrystalline $\mathrm{CeCu}_{4} \mathrm{Al}$ up to $200 \mathrm{~K}$. (b) Low temperature $C / T$ dependence as a function of $T^{2}$, used to determine a $\gamma$ value.

However, we have observed a maximum at about $2.5 \mathrm{~K}$ in $C(T)$ dependence. Therefore, to study the origin of this maximum we have measured the influence of 
magnetic fields on the low temperature part of $C(T)$ of $\mathrm{CeCu}_{4} \mathrm{Al}$. In Fig. 2 this behaviour is presented for magnetic fields up to $9 \mathrm{~T}$. We observed that above $20 \mathrm{~K}$ there is no important influence of the magnetic field on $C(T)$. Below $20 \mathrm{~K}$ and above maximum, one could see the increasing magnitudes with increasing magnetic fields. Below maximum the situation is opposite. The maximum itself is shifting to higher temperatures with increasing magnetic fields. The magnetic field does not influence the $\gamma$ value determined for $T>11 \mathrm{~K}$. The $C / T$ extrapolation for lowest temperatures decreases with increasing magnetic field as in [3]. However, small maximum appears above $4 \mathrm{~T}$. At $9 \mathrm{~T}$ the extrapolated $\gamma$ value from maximum yields to a $0.73 \mathrm{~J} \mathrm{~mol}^{-1} \mathrm{~K}^{-2}$, while lowest temperatures extrapolation yields to $0.55 \mathrm{~J} \mathrm{~mol}^{-1} \mathrm{~K}^{-2}$.

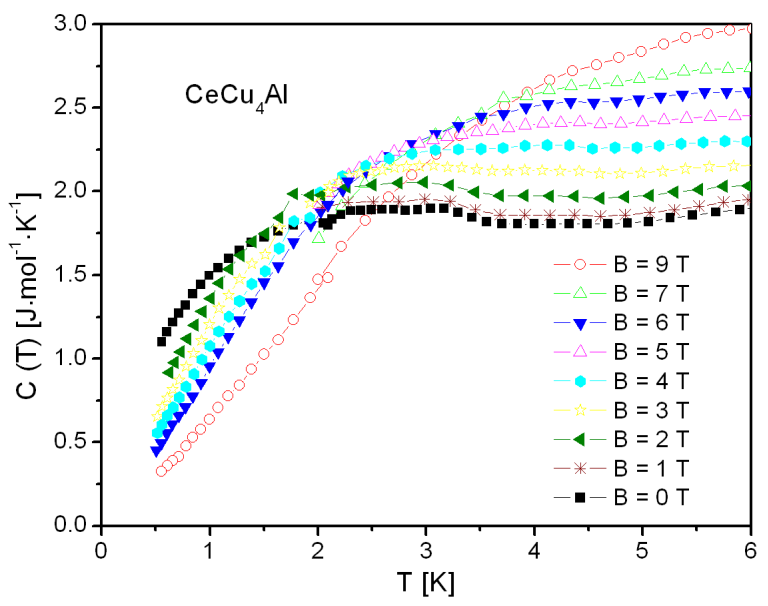

Fig. 2. The low temperature part of the heat capacity $C(T)$ of the polycrystalline $\mathrm{CeCu}_{4} \mathrm{Al}$ in the applied magnetic fields up to $9 \mathrm{~T}$.

In Fig. 3a the temperature dependence of magnetic susceptibility $\chi^{-1}(T)$ is presented. The measurements did not reveal the magnetic transition. Above about $40 \mathrm{~K} \chi^{-1}(T)$ follows a Curie-Weiss law with the effective magnetic moment $\mu_{\text {eff }}=$ $2.54 \mu_{\mathrm{B}}$ and the paramagnetic Curie temperature $\theta_{\mathrm{p}}=-10 \mathrm{~K}$. The experimental value of $\mu_{\text {eff }}$ is equal to the calculated one for a free $\mathrm{Ce}^{3+}$ ion $\mu_{\text {eff }}=g[j(j+$ $1)]^{-1 / 2}=2.54 \mu_{\mathrm{B}}$, thus indicating the presence of well localized magnetic moments carried by the stable $\mathrm{Ce}^{3+}$ ions. At low temperatures the $\chi^{-1}(T)$ curve deviates from the straight-line behaviour. This deviation correlates with the maxima in $C(T)$. Therefore, we suppose their probable crystal electric-field (CEF) origin like Schottky anomaly.

The magnetic field dependences of magnetisation are presented in Fig. 3b. The temperature evolution confirms the previous observation and the absence of magnetic ordering down to $2 \mathrm{~K}$. From this we conclude that $\mathrm{CeCu}_{4} \mathrm{Al}$ is in the heavy fermion state at low temperature. The magnetic field behaviour of $C(T)$, 

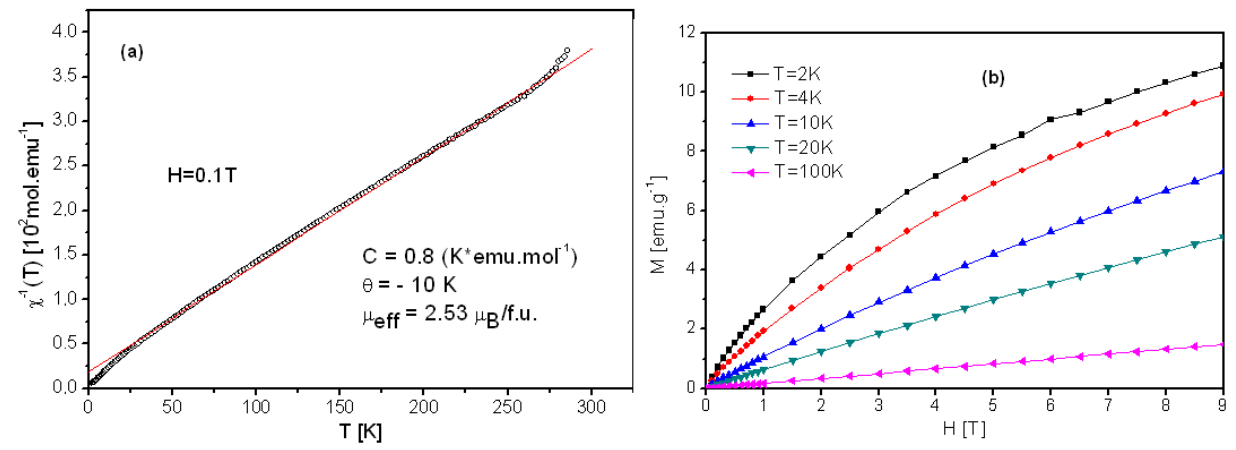

Fig. 3. (a) The magnetic susceptibility $\chi^{-1}(T)$ of $\mathrm{CeCu}_{4} \mathrm{Al}$. (b) Magnetization $M(H)$ of $\mathrm{CeCu}_{4} \mathrm{Al}$ for different temperatures.

$\chi^{-1}(T), M(T)$ and the maximum in $C(T)$ at $2.5 \mathrm{~K}$ hints to the probable presence of antiferromagnetic impurities.

In conclusion, we have measured the heat capacity of polycrystalline $\mathrm{CeCu}_{4} \mathrm{Al}$ as function of an applied magnetic field. We observe typical heavy fermion behaviour with $\gamma$ value of about $210 \mathrm{~mJ} \mathrm{~mol}^{-1} \mathrm{~K}^{-2}$. The $C / T$ extrapolation for lowest temperatures decreases with increasing magnetic field as in [3]. At $9 \mathrm{~T}$ the extrapolated $\gamma$ value from maximum yields to a $0.73 \mathrm{~J} \mathrm{~mol}^{-1} \mathrm{~K}^{-2}$, while lowest temperatures extrapolation yields to $0.55 \mathrm{~J} \mathrm{~mol}^{-1} \mathrm{~K}^{-2}$. We have observed at about $2.5 \mathrm{~K}$ a maximum in $C(T)$ dependence. The measurements of the magnetic susceptibility confirm the observations derived from the heat capacity. Above about $40 \mathrm{~K} \chi^{-1}(T)$ follows a Curie-Weiss law with the effective magnetic moment $\mu_{\mathrm{eff}}=2.54 \mu_{\mathrm{B}}$ and the paramagnetic Curie temperature $\theta_{\mathrm{p}}=-10 \mathrm{~K}$.

\section{Acknowledgments}

This work was supported partly by the COST-ECOM P16, by Science and Technology Assistance Agency - APVT-51-031704, by VEGA 6165, by the contract CE of SAS. J.S. and E.S. are grateful for support of GACR 106/06/0368.

\section{References}

[1] E. Bauer, D. Gignoux, D. Schmitt, K. Winzer, J. Magn. Magn. Mater. 69, 158 (1987).

[2] E. Bauer, E. Gratz, N. Pillmayr, Solid State Commun. 62, 271 (1987).

[3] B. Andraka, J.S. Kim, G.R. Stewart, Z. Fisk, Phys. Rev. B 44, 4371 (1991). 